\title{
Anticipated Support From Neighbors and Physical Functioning During Later Life
}

\author{
BENJAMIN A. SHAW \\ State University of New York at Albany
}

\begin{abstract}
This study has two main objectives: (1) to assess age variations in perceived support from neighbors among a nationally representative sample of adults aged 25 to 74 and (2) to examine the association between anticipated support from neighbors and physical functioning within a subsample of older adults. The findings suggest that anticipated support from neighbors is stronger among older adults, primarily because of more frequent contact with neighbors and residential stability. Within the older subsample, an inverse association between perceived support from neighbors and functional limitations is evident. Further analyses show that this association is strongest among those with infrequent contact with family members. No differences in this association were found with respect to marital status. Taken together, it appears that anticipated support from neighbors facilitates the maintenance of functional ability among some older adults. Interventions aiming to promote successful aging by enhancing this source of support should be developed and evaluated.
\end{abstract}

Keywords: social support; neighbors; functional disability; elderly

Knowledge of the protective effects of supportive social relationships has advanced steadily during the past several years. Persuasive evidence showing the physical and mental health benefits of receiving social support and being socially integrated continues to accumulate (Eng et al. 2002; Seeman, Lusignolo, and Albert 2001). Particularly compelling from an intervention standpoint are findings from the gerontological literature indicating that simply knowing that support is available from significant others should the need arise (i.e.,

AUTHOR'S NOTE: This research was funded by the National Institutes of Health, National Institute on Aging, Grant No. P01AG020166, "Integrative Pathways to Health and Illness." Address all communications to Benjamin A. Shaw, Department of Health Policy, Management, and Behavior, School of Public Health, University at Albany, One University Place, Rensselaer, NY 12144-3456; phone: (518) 402-0325; fax: (518) 402-0414; e-mail: bashaw @ albany.edu.

RESEARCH ON AGING, Vol. 27 No. 5, September 2005 503-525 DOI: $10.1177 / 0164027505277884$

() 2005 Sage Publications 
anticipated or perceived support) may be more beneficial to health and well-being than actually receiving support or maintaining a large number of social ties (Krause 1997; Wethington and Kessler 1986). This research suggests that a key objective in promoting successful aging ought to be improving the perceived strength of one's "social safety net" and not just increasing the amount of support one actually receives or modifying the structural characteristics (e.g., size or frequency of contact) of one's social network (Krause 2001).

Although the findings from this research represent important advances, they also make apparent a critical gap in the current study of social relationships and health. In particular, much of the current research in this area focuses on the health benefits of social support that is perceived to be available from the entire social network taken as a whole. It is important to recognize, however, that because the social networks of adults are likely to include a remarkably diverse set of potential sources of support-ranging from the most intimate of ties to those of a far less intimate nature-we must learn more about the source-specific impact of anticipated social support. In this regard, research on the health benefits of family relationships has led to a common appreciation of the importance of perceived support from intimate social ties (Ross, Mirowsky, and Goldsteen 1990). However, we currently know far less about the health impact of anticipated support from less intimate social ties. The purpose of the present study is to examine the potential health effects of anticipated social support from one relatively understudied and nonintimate source: neighbors (Wethington and Kavey 2000). A comprehensive understanding of the relationship between anticipated support from neighbors and health may point to new opportunities for community-based efforts to promote successful aging.

To clarify the potential association between anticipated support from neighbors and health, four issues are addressed in the discussion that follows. First, the theoretical rationale justifying the health benefits of anticipated support from network members as a whole is offered. Next, the possible benefits of anticipated support from neighbors, in particular, are briefly reviewed. Following this, the potentially unique placement of neighbors within the social networks of older adults is considered. Finally, the proposition that older adults who lack key interpersonal relationships may derive particularly profound benefits from supportive neighbors is presented. 


\section{Anticipated Support and Health}

Anticipated support is defined as the belief that others will provide assistance should the need arise (Wethington and Kessler 1986). As an indicator of positive social relations and a determinant of health and physical functioning, this construct appears to have advantages over measures of received support and social embeddedness for two related reasons. First, recognizing that others are available to help provides individuals with a "social safety net," which encourages efforts to resolve problems autonomously, thereby promoting health-enhancing feelings of personal control and self-worth and preserving independence (Krause 2001; Rodin 1990). In contrast, while receiving assistance from network members may be beneficial up to a certain point, receiving excessive amounts of support may actually compromise an individual's sense of personal competence and well-being (Silverstein, Chen, and Heller 1996).

In addition, anticipated support may be a better predictor of health and physical functioning than measures of received support and social embeddedness because it is a more valid indicator of the presence of a supportive social network. For instance, a low level of received support is not necessarily a valid indicator of an inadequate support system because this may actually indicate a current lack of need for support from network members because of strong personal coping resources (Eckenrode and Wethington 1990). In addition, measures of social embeddedness, which merely quantify the size of one's network of social ties and frequency of contact with network members, fail to reveal the degree of supportiveness of one's social network. In contrast, measures of anticipated support seem to provide an accurate representation of the supportiveness of one's network as they are likely to reflect both the support that has been received in the recent past and the support that is currently not needed but is readily available (Wethington and Kessler 1986).

Despite the advantages of this form of support, its potential impact on physical functioning among older adults has not been adequately studied. Most of the current research on social relationships and physical functioning among older adults has focused on the impact of social network characteristics or received support (e.g., Avlund et al. 2004; Giles et al. 2004). Although there is some recent evidence of an association between anticipated support, in general, and functional 
status (Shaw and Janevic 2004), studies focusing specifically on the potential benefits of anticipated support from social ties outside of the intimate social network, such as neighbors, have been extremely rare. Therefore, focusing specifically on the role of neighbors in older adults' social safety nets should provide for a more detailed and complete understanding of the health and functional benefits of anticipated support.

\section{The Benefits of Anticipated Support From Neighbors}

In recent years, there has been a resurgence of interest in the relationship between neighborhood characteristics and health (e.g., Balfour and Kaplan 2002; Kawachi and Berkman 2003). Research on the role of social support from neighbors, however, dates back at least two decades (Cantor 1979). This work suggests that support from neighbors fulfills a crucial need for many individuals by supplementing the support provided by more intimate network members. For instance, neighbor support may serve to compensate in a variety of ways for a lack of support from more intimate ties when support from these ties is not readily available (Cantor 1979; Rook and Schuster 1996). Others suggest that support from neighbors may serve not only a compensatory role but also a task-specific role (Litwak 1985). That is, support from neighbors may be beneficial primarily because neighbors may be the network members in the best position to respond to specific types of problems or tasks, such as those requiring geographic proximity.

What this research does not fully address, however, is the extent to which anticipated support from neighbors may actually promote health and physical functioning. To understand the potential benefits of anticipated support from neighbors, it is first necessary to consider how actual support from neighbors may protect against functional disability within the aging population (Wethington and Kavey 2000). For instance, support from neighbors may come in the form of monitoring one's health and safety. The geographic proximity of neighbors should enable them to notice when something may be wrong with an individual (e.g., an injury because of a fall) and respond quickly to signs of need, perhaps preventing the escalation of problems that could lead to functional disability. In addition, a supportive network 
of neighbors should facilitate the exchange of tangible assistance when needed, such as transportation or assistance with household maintenance. Ready access to such tangible assistance should directly enhance an individual's ability to perform activities of daily living. Strong ties with neighbors may also protect against functional decline by providing opportunities for social activity and engagement (Everard et al. 2000).

Although actually experiencing each of these forms of neighborly support and interaction should help to protect against functional decline, simply anticipating access to this type of support from neighbors should also be protective. This is because perceiving that one's safety is being monitored, that tangible assistance is available if needed, and that opportunities for social engagement exist should provide individuals with a strong sense of security. Such a sense of security is expected to enhance physical functioning by bolstering feelings of personal control that empower individuals to maintain an active and independent lifestyle in select domains (Baltes 1996).

\section{Aging and Anticipated Support From Neighbors}

Although adults of all ages stand to benefit from having supportive neighbors, older adults may reap the greatest benefits because perceptions of access to support from neighbors are expected to increase with age. An age-related increase in anticipated support from neighbors is expected for several reasons. First, compared with younger adults, older adults are more likely to have stable residences. For example, during a given period, older adults are less likely than younger adults to move from one neighborhood to another (Burkhauser, Butrica, and Wasylenko 1995). This means that older adults are likely to be more firmly established in their neighborhoods, and consequently, more likely to have formed long-standing, supportive relationships with their neighbors (Fisher 1982).

In addition, older adults may have more time than younger adults to develop supportive social ties with neighbors. With advancing age, time spent working for pay decreases sharply (Gauthier and Smeeding 2003). At least some of this extra time may be spent cultivating neighborly ties. 
Finally, anticipated support from neighbors may increase with advancing age to the extent that older adults actively select neighbors to become increasingly prominent members of their narrowing social networks. According to socioemotional selectivity theory (Carstensen 1991), as a strategy for successful aging, adults entering later life seek to maximize the efficiency of their social relationships by devoting the bulk of their social resources to maintaining primarily the most intimate social ties. The underlying logic behind this social transition is that as individuals get older and begin to perceive that time is limited, short-term rather than long-term goals become increasingly important. Emotional well-being is thought to be one of the most salient of short-terms goals for older adults, and maintaining intimate social ties appears to offer the best opportunities for satisfying this particular goal (Baltes and Carstensen 1999).

Following the same basic logic used in selectivity theory, however, it is also possible to view maintaining ties with neighbors as an efficient way for older adults to meet other short-term needs, such as safety monitoring, tangible assistance, and social engagement. The easy accessibility of neighbors appears to make them particularly well suited for satisfying these other immediate and ongoing needs of older adults. For this reason, in addition to striving to maintain a network of close interpersonal ties, older adults may also begin to rely more heavily on neighbors for support than do young adults.

\section{Compensating for the Lack of Intimate Ties}

Even though neighborly ties are expected to increase with age, it is important to recognize that anticipated support from neighbors may not be an equally beneficial resource for all aging adults. For instance, the presence or absence of more intimate interpersonal relationships may account for variation in the effects of support from neighbors on physical functioning in later life. Specifically, it is expected that support from neighbors may be particularly valuable to the health of individuals with a depleted intimate social network, such as those who are not married or those who have little contact with family members (Rook and Schuster 1996). This is consistent with the hierarchicalcompensatory model of support seeking (Cantor 1979). According to this model, most people prefer to receive support from intimate part- 
ners. Only when these preferred sources of support are absent can support from another source, such as a neighbor, effectively compensate. The health effects of this hierarchical substitution of support involving neighbors, however, have not been adequately examined (Wethington and Kavey 2000).

Viewed broadly, this theoretical discussion highlights the potential importance of anticipated support from neighbors as a resource for protecting against functional impairment among older adults and as a possible target for promoting successful aging. Accordingly, the following hypotheses emerge from this discussion:

Hypothesis 1: Levels of anticipated support from neighbors increase with age, primarily because of older adults having greater residential stability and more interaction with their neighbors.

Hypothesis 2: Individuals reporting high levels of anticipated support from neighbors have fewer functional limitations than those reporting low levels of anticipated support.

Hypothesis 3: The functional benefits associated with anticipated support from neighbors are at least partially mediated by feelings of personal control.

Hypothesis 4: During later life, older adults who are unmarried and have infrequent contact with family members benefit the most from anticipated neighbor support.

\section{Method}

\section{SAMPLE}

The data for this study come from the National Survey of Midlife Development in the United States (MIDUS), 1995-1996 (Brim et al. 1996). Participants are a nationally representative random-digit-dial sample of noninstitutionalized, English-speaking adults, aged 25 to 74, residing in the contiguous United States. Older adults and men were oversampled. All of the analyses for this study are based on weighted data. The data were collected via an initial telephone interview and a follow-up mailed questionnaire, both of which were completed in 1995. The estimated response rate for MIDUS was $60.8 \%$ (Brim et al. 1996), resulting in a weighted sample size of 3,044. 
TABLE 1

Descriptive Statistics for Major Study Variables

\begin{tabular}{lcc}
\hline & \multicolumn{2}{c}{ M (SD) or \% } \\
\cline { 2 - 3 } & $\begin{array}{c}\text { Full sample } \\
\text { Age 25 to } 74)\end{array}$ & $\begin{array}{c}\text { Older subsample } \\
\text { (Age 50 to 74) }\end{array}$ \\
Predictor (range or coding) & $\mathrm{N}=2,913$ & $\mathrm{n}=943$ \\
\hline Age & $45.09(13.53)$ & $60.56(7.06)$ \\
Marital status $(1=$ married) & $74.60 \%$ & $73.24 \%$ \\
Education (1-12) & $6.24(2.41)$ & $5.81(2.48)$ \\
Race (1 = White) & $82.82 \%$ & $87.85 \%$ \\
Sex $(1=$ male) & $43.39 \%$ & $41.74 \%$ \\
Anticipated neighbor support (1-4) & $3.39(0.84)$ & $3.55(0.75)$ \\
Neighbor contact (1-6) & $4.97(1.28)$ & $5.08(1.25)$ \\
Years in neighborhood (0-73) & $12.41(13.01)$ & $19.84(15.16)$ \\
Rent or own home (1 = rent) & $25.01 \%$ & $12.86 \%$ \\
Family contact (1-8) & - & $5.97(1.51)$ \\
Personal control beliefs (8-56) & - & $41.09(11.13)$ \\
Functional limitations $(0-27)$ & - & $6.82(7.22)$ \\
\hline
\end{tabular}

For the analyses assessing the association between age and anticipated support from neighbors, all available data are used. For all other analyses, data from only those respondents aged 50 and above are used. Table 1 displays descriptive statistics for all of the major study variables used in each of these sets of analyses. Listwise deletion of missing values is used to deal with item nonresponse, resulting in the loss of approximately $5 \%$ of cases from the full sample and $11 \%$ of cases from the older subsample. Approximately $43.39 \%$ of the full sample is male, and $82.82 \%$ is White. The older subsample has a greater percentage of women and White respondents. The average age of participants was $45.09(S D=13.53)$ in the full sample and 60.56 $(S D=7.06)$ in the older subsample. Almost three quarters $(74.99 \%)$ of respondents in the full sample own their home, whereas $87.14 \%$ of the older subsample are home owners. Respondents in the full sample report having lived in their current neighborhood for an average of $12.41(S D=13.01)$ years. This compares to an average length of residence of $19.84(S D=15.16)$ years in the older subsample. 
The major independent variable in this study is anticipated support from neighbors. This construct is measured with a single item taken from a scale measuring perceived neighborhood quality in the MIDUS survey. Specifically, respondents were asked the degree to which the following statement describes their situation: "I could call on a neighbor for help if I needed it." Response options range from 1 (a lot) to 4 (not at all). This item is recoded so that higher scores reflect more anticipated support. ${ }^{1}$

The major dependent variable in this study, physical health status, is assessed with nine widely used indicators of functional limitations (Katz et al. 1963; Nagi 1976; Rosow and Breslau 1966). Specifically, respondents rated how much their health limited them in performing the following nine activities of daily living: lifting or carrying groceries; bathing or dressing yourself; climbing several flights of stairs; bending, kneeling, or stooping; walking more than a mile; walking several blocks; walking one block; vigorous activity; moderate activity. Response options ranged from 0 (not at all) to 3 (a lot), and all nine items were summed so that higher scores reflect more functional limitation. The internal consistency reliability estimate for this scale is .93 .

Several intervening variables are also assessed in this study. Personal control beliefs are measured using five items from Pearlin and Schooler's (1978) Mastery Scale, plus three additional items. Respondents were asked to indicate their agreement with the following statements: "I have little control over things that happen to me"; "There is really no way I can solve the problems I have"; "I sometimes feel I am being pushed around in my life"; "There is little I can do to change the important things in my life"; "I often feel helpless in dealing with the problems of life"; "Other people determine most of what I can and cannot do"; "What happens in my life is beyond my control"; and "There are many things that interfere with what I want to do." Responses to each item were scored on a 7-point scale ranging from 1 (agree strongly) to 7 (disagree strongly). An index was constructed by adding together the scores for all eight items; higher scores reflect a greater sense of personal control. The internal consistency reliability estimate for this scale is .87 . 
Frequency of family contact is measured with the following question from the MIDUS survey: "How often are you in contact with any members of your family - that is, any of your brothers, sisters, parents or children who do not live with you-including visits, phone calls, letters, or electronic mail messages?" Response options included 1 (never or hardly ever), 2 (less than once a month), 3 (about once a month), 4 (2 or 3 times a month), 5 (about once a week), 6 (several times a week), 7 (about once a day), and 8 (several times a day). Neighbor contact is an indicator of the frequency of interaction with neighbors and is measured with a 6-point Likert-type scale ranging from 1 (never or hardly ever) to 6 (almost every day). Residential stability is measured with two variables. Years in neighborhood reflect the number of years a respondent has lived in his or her current neighborhood or township. Rent or own home is coded in the following binary format ( $1=$ rent, $0=$ own or pay mortgage).

Other key variables used in the current study include age, which is scored continuously in years, and marital status $(1=$ married or living with a partner, $0=$ other). A select set of variables are also included as statistical controls in the multivariate analyses. These variables are sex $(1=$ male, $0=$ female $)$; race $(1=$ White, $0=$ other $)$; and education, which reflects the highest grade or year of schooling completed and is coded with a 12-point ordinal scale ranging from 1 (no school or some grade school) to 12 (doctoral or other professional degree).

\section{DATA ANALYSIS PLAN}

The analyses for this study use ordinary least squares (OLS) multiple regression and proceed in three main stages. The intitial stage of analysis addresses Hypothesis 1 in two steps. First, the association between age and anticipated support from neighbors is estimated, after controlling for the effects of gender, race, education and marital status. Next, the variables neighbor contact, years in neighborhood, and rent or own are added to the model to determine the degree to which they account for the association between age and anticipated support from neighbors.

In the second stage of the analysis, Hypotheses 2 and 3 are addressed. First, within the older subsample, the association between 
anticipated support from neighbors and functional limitations is estimated after controlling for some basic demographics and other neighborhood-related characteristics (e.g., neighbor contact, years in neighborhood, and rent or own home). Controlling for these neighborhood characteristics is necessary to isolate the unique association between anticipated support from neighbors and functional limitations by accounting for other potential influences on functional status related to interaction with neighbors and residential stability.

After estimating the main effect of anticipated support from neighbors, the variable personal control beliefs is included in the model. By noting the degree to which the main effect of anticipated support from neighbors changes as a result of this step, it is possible to determine the extent to which a sense of personal control accounts for, or mediates, the association between anticipated support from neighbors and functional limitations.

The final stage of analysis addresses Hypothesis 4. In particular, the purpose of this stage is to determine if the association between anticipated neighbor support and functional limitations differs between those who are married and unmarried or between individuals with different levels of contact with family members. These analyses are conducted by entering the following interaction terms to the model estimating the association between anticipated support from neighbors and functional limitations: Anticipated Support From Neighbors $\times$ Marital Status and Anticipated Support From Neighbors $\times$ Frequency of Family Contact. If a coefficient associated with an interaction term is statistically significant, this means that the relationship between anticipated neighbor support and functional limitations varies depending on the presence of more intimate social ties. To clarify the findings associated with a significant interaction term, a formula provided by Aiken and West (1991) is used to estimate and test the significance of the association between anticipated neighbor support and functional limitations at various levels of marital status or family contact. If these interaction effects are consistent with the hierarchicalcompensatory model, then the proposed inverse association between neighbor support and functional limitations should appear strongest among respondents who are not married as well as those who have infrequent contact with family members. 
TABLE 2

Regression of Anticipated Support From Neighbors on Age and Other Demographic and Neighborhood-Related Variables $(N=2,913)$

\begin{tabular}{llllll}
\hline & \multicolumn{2}{c}{ Column $A$} & & \multicolumn{2}{c}{ Column $B$} \\
\cline { 2 - 3 } \cline { 5 - 6 } Predictor & $\beta$ & $\mathrm{B}$ & & $\beta$ & $\mathrm{B}$ \\
\hline Age & $.185^{* * *}$ & .011 & & $.070^{* * *}$ & .004 \\
Marital status (1 = married/partnered) & $.103^{* * *}$ & .198 & & $.037^{*}$ & .072 \\
Education & .023 & .008 & & .020 & .007 \\
Race (1 = White) & $.039^{*}$ & .086 & & $.045^{* *}$ & .100 \\
Sex (1 = male) & $-.071^{* * *}$ & -.120 & & $-.086^{* * *}$ & -.145 \\
Neighbor contact & & & & $.347^{* * *}$ & .227 \\
Years in neighborhood & & & & $.066^{* *}$ & .004 \\
Rent or own home (1 $=$ rent) & & & & $-.184^{* * *}$ & -.357 \\
$R^{2}$ & .051 & & .211 & \\
\hline
\end{tabular}

$* p<.05 . * * p<.01 . * * * p<.001$.

Results

\section{AGE AND ANTICIPATED SUPPORT FROM NEIGHBORS}

The association between age and anticipated support from neighbors is shown in Column A of Table 2. As expected, age is positively associated with anticipated support from neighbors $(\beta=.185, p<$ $.001)$. This means that, on average, levels of anticipated support from neighbors are higher among older adults.

In Column B of Table 2, the association between age and anticipated support from neighbors remains significant, but the effect size is much smaller $(\beta=.070, p<.001)$. This decline in the effect size is the consequence of adding the variables neighbor contact, years in neighborhood, and rent or own home to the model. Therefore, it can be concluded that these three variables explain approximately 62\% (1-.070/ $.185)$ of the association between age and anticipated support from neighbors.

Other important findings regarding predictors of anticipated support from neighbors also appear in Table 2. For example, it should be noted that an age effect is apparent even after controlling for the effects of these mediating variables. In other words, regardless of the frequency of interaction with neighbors or residential stability, older adults appear to report more anticipated support from neighbors than do younger adults. In addition, adults who are married report higher 
levels of anticipated support from neighbors than do unmarried adults $(\beta=.037, p<.05)$, Whites report more anticipated support from neighbors than do non-Whites $(\beta=.045, p<.01)$, and men report lower levels of anticipated support from neighbors than do women $(\beta=-.086, p<.001)$. Furthermore, those who rent their homes report less anticipated support from neighbors than do home owners $(\beta=$ $-.184, p<.001$ ), and levels of neighbor support are positively associated with frequency of contact with neighbors $(\beta=.347, p<.001)$ and the number of years one has lived in his or her current neighborhood $(\beta=.066, p<.01)$.

\section{ANTICIPATED SUPPORT FROM NEIGHBORS} AND FUNCTIONAL LIMITATIONS

Table 3 displays findings regarding the association between anticipated support from neighbors and functional limitations. Column A of this table shows the findings from a model that includes only the control variables and anticipated support from neighbors as predictors. As should be expected, relatively high levels of functional impairment are associated with being older $(\beta=.172, p<.001)$. Protection against functional impairment is associated with being male $(\beta=-.141, p<.001)$ and having a high level of education $(\beta=-.148, p<.001)$. Functional impairment is not associated with frequency of contact with neighbors, number of years in one's current neighborhood, or renting/owning one's home. This model, however, does show an inverse association between anticipated support from neighbors and functional limitations $(\beta=-.110, p<.01)$. Individuals who report relatively high levels of anticipated neighbor support tend to have fewer functional limitations than those who perceive less access to support from neighbors. Together, these variables explain about $10.9 \%$ of the variance in functional limitations.

In Column B, the variable personal control beliefs is added to the model. The findings from this model show that respondents who report having a strong sense of control have relatively few functional limitations $(\beta=-.209, p<.001)$. The mediating effect of this variable can be assessed by observing how the association between anticipated support from neighbors and functional limitations changes between Columns A and B. The findings from Table 3 show that the association between anticipated support from neighbors and functional limita- 


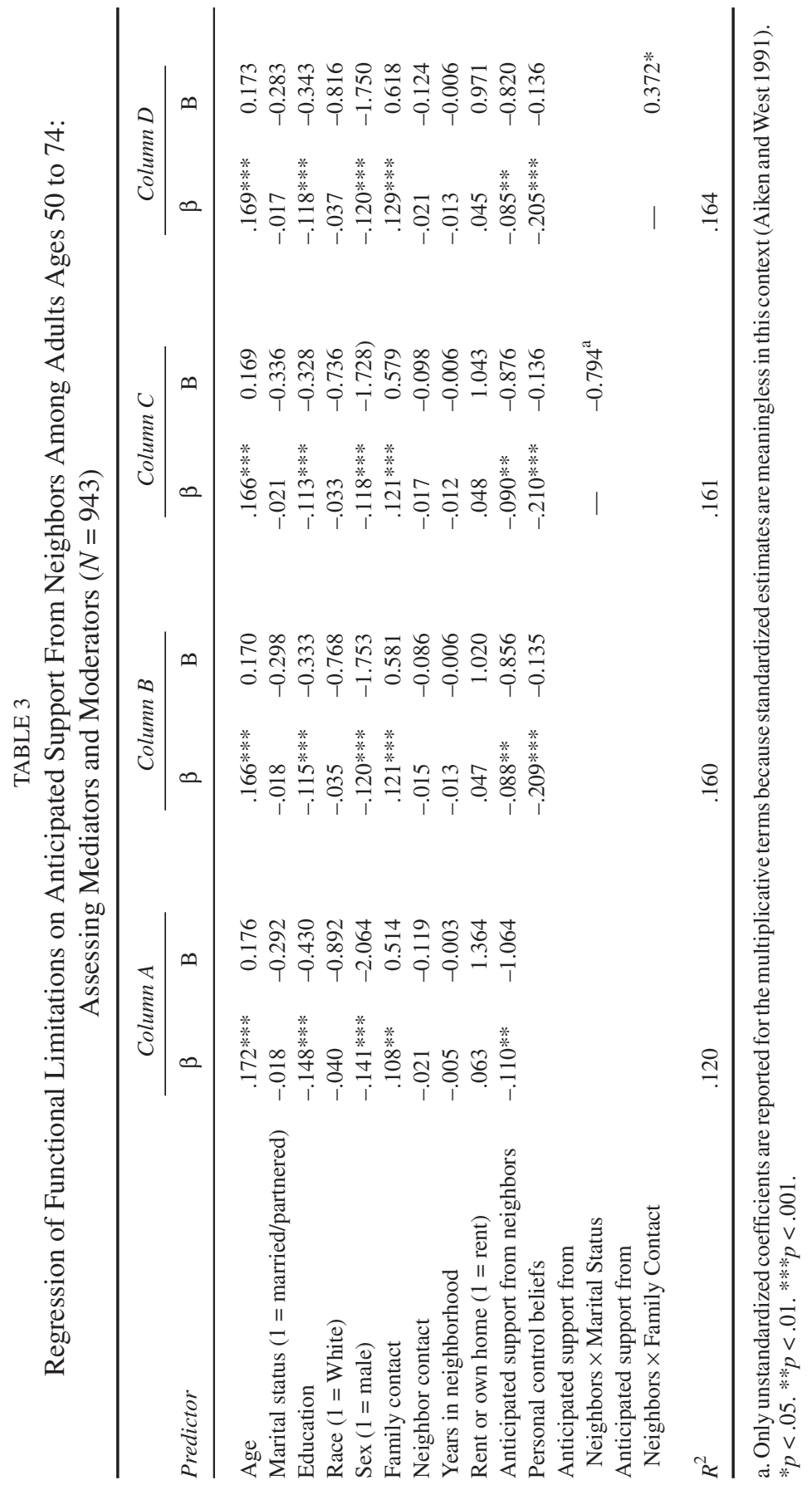


tions declines by $20 \%$ (from - .110 to -.088 ) when personal control beliefs are accounted for. This means that personal control beliefs explain approximately one fifth of the association between anticipated support from neighbors and functional limitations.

The final two columns of this table present the results of the models testing the modifying effects of marital status and family contact on the association between anticipated neighbor support and functional limitations. Column $\mathrm{C}$ shows the findings for the interaction between anticipated support from neighbors and marital status. The coefficient representing this interaction term is not significant. This suggests that the association between anticipated support from neighbors and functional status does not differ between older adults who are married and those who are not.

Column D shows the findings for the interaction between anticipated support from neighbors and frequency of family contact. The coefficient representing this interaction term is statistically significant $(b=.372, p<.05)$. This suggests that the association between anticipated support from neighbors and functional limitations varies across different levels of family contact.

The specific nature of this variation can be determined by calculating a series of simple slopes for the association between anticipated neighbor support and functional limitations at select levels of family contact (for a complete description of this procedure, see Aiken and West 1991:12-17). Table 4 presents simple slopes associated with monthly contact, weekly contact, and daily contact with any family member. This table shows that among those with daily contact with family members, there is no significant association between levels of anticipated neighbor support and functional limitations $(\beta=-.045)$. However, among those reporting weekly contact with family members, high levels of anticipated support from neighbors are associated with low levels of functional limitations $(\beta=-.123, p<.001)$. Furthermore, among those who are in contact with family members on only a monthly basis, there appears to be an even stronger inverse association between neighbor support and functional limitations $(\beta=-.200, p<$ .001). Taken together, these results support the study's fourth hypothesis by suggesting that the association between anticipated neighbor support and functional limitations is strongest among older adults who have the least contact with family members. 
TABLE 4

Impact of Anticipated Support From Neighbors on Functional Limitations at Various Levels of Family Contact

\begin{tabular}{llc}
\hline & \multicolumn{2}{c}{ Effect on Functional Limitations } \\
\cline { 2 - 3 } Family Contact & \multicolumn{1}{c}{$\mathrm{B}$} \\
\hline "About once a day" & -.045 & -.437 \\
"About once a week" & $-.123^{* * *}$ & -1.181 \\
"About once a month" & $-.200^{* * *}$ & -1.925 \\
\hline
\end{tabular}

$* * * p<.001$

\section{Discussion}

These findings largely support the major hypotheses and theoretical rationale of this study and highlight the benefits that older adults may derive from anticipating access to support from neighbors. In fact, the findings from this study not only suggest that older adults may derive important benefits from anticipated support from neighbors; they also suggest that older adults are more likely to perceive high levels of support from neighbors compared to younger adults. The association between age and anticipated neighbor support appears to be mainly due to a higher frequency of contact with neighbors and greater residential stability among older adults. Although these findings may be simply due to cohort effects, they could also be interpreted as an indication that neighbors are an increasingly important resource for aging adults.

The geographic proximity of neighbors is likely to enable them to fulfill many of the immediate or short-term needs that an older adult may have, such as monitoring of safety, tangible assistance, and social engagement. As a result, perceiving that people within one's immediate environs could be called upon for assistance if necessary is likely to provide older adults with a sense of security about being able to survive future predicaments, such as an illness or injury (Ross and Mirowsky 2002). Feeling the security of a readily available "social safety net" is likely to encourage attempts to address daily challenges autonomously (Wethington and Kessler 1986). Successfully coping with challenges bolsters feelings of personal control (Rodin 1990), which may then motivate elders to remain physically active and independent and help protect against the incidence and progression of 
physical disability (Gill et al. 1994; Mendes de Leon et al. 1996). In contrast, the lack of a readily available social safety net may erode feelings of personal control, leading to increasing inactivity and ultimately the loss of functional ability (Simonsick et al. 1993). Overall, personal control beliefs account for $20 \%$ of the association between anticipated support and functional impairment.

The apparent benefits of anticipated support from neighbors may be especially important given the current trends in the geographic mobility of families. According to Pillemer and Glasgow (2000), current trends in family mobility, such as the out-migration of young adults from rural areas and the migration of "young-old" adults to warm climates, have resulted in substantial segments of the older adult population living in the absence of nearby close relatives. This lack of geographic proximity with relatives seems to reduce opportunities for social contact between older adults and relatives such as adult children (Krout 1988) and thus presents the need for alternative resources for helping to meet one's immediate needs for assistance and social interaction. The current findings are consistent with this idea in that they show that anticipated support from neighbors is associated with the most benefits (and the lack of support is associated with the most harm) among older adults who are in contact with their family members on no more than a weekly basis.

Apparently, however, a similar logic regarding the compensatory nature of support from neighbors may not hold true when considering marital status. Although one might expect that support from neighbors would be particularly important among older adults who are not married, the current findings do not support this hypothesis. According to the current findings, anticipated support from neighbors is associated with better physical functioning among both married and unmarried older adults. Perhaps what this suggests is that anticipated support from neighbors fulfills a task-specific, rather than a compensatory, role with respect to marital status (Litwak 1985). That is, perhaps support from neighbors is important to both married and unmarried older adults because neighbors offer unique types of assistance that are beneficial regardless of whether or not a spouse is present. In addition, it is possible that the influence of neighborly support is more limited than expected among unmarried older adults because these individuals may tend to rely on sources other than their neighbors (e.g., adult children) for actual support and assistance or may be less 
likely than their married counterparts to call upon their neighbors during times of need, perhaps due to a lack of relational competence or simply not wanting to impose (Carpenter 1993).

Despite the remaining questions that these findings present, taken as a whole, it appears that access to support from neighbors is an important resource for at least some groups of older adults. As such, the practical implications of these findings must be considered. For example, the current findings seem to indicate the potential effectiveness of efforts to develop neighborhood support networks and increase elders' awareness of neighbors who may be able to offer assistance during times of need. Specifically, the current findings suggest that such programs could have a substantial public health impact in communities by delaying functional decline among the elderly and thus facilitate their ability to remain living in the community (for an example of such a program, see www.caringneighborhoods.org).

Identifying the conditions that best foster the development of neighborhood social ties and the perception of support availability within neighborhoods, however, is an important question for future research. Current thinking suggests that the social and physical characteristics of communities could play a substantial role. For example, social cohesion and mutual trust within a community are likely to have profound influences on social interaction among neighbors (Sampson 2003). To the extent that progress can be made to improve the degree of social cohesion and trust within the communities in which older adults live, establishing neighborhood support networks to promote successful aging should become easier. The role of the built environment, and specifically the role of senior housing, as an agent of social capital and cohesion is a growing area of research (Cannuscio, Block, and Kawachi 2003).

Before delving too far into efforts to develop neighborhood support networks for aging adults, however, more research needs to be conducted to confirm and clarify the current findings regarding the health effects of anticipated support from neighbors. Important limitations of the current study hamper one's ability to make definitive conclusions about these effects. For example, in the current study, anticipated support from neighbors is represented with only a single-item measure. It should be noted that a single-item measure of a construct such as this is subject to random measurement error and thus low reliability. Although this type of error only attenuates bivariate associa- 
tions, thus creating a conservative estimate of the health effects of support from neighbors, in multivariate analyses, the consequences of measurement error are less predictable. Future research looking into the potential health impact of perceived support from neighbors must strive to employ a more reliable measure of this construct. Moreover, a more comprehensive measure of anticipated support from neighbors would help guard against the possibility that unmeasured social network characteristics (e.g., social integration) are being represented and would also allow for a more fine-grained analysis of the differential effects of various types of anticipated support (e.g., emotional and tangible) from neighbors.

Another limitation of this study stems from its cross-sectional nature. In particular, data on anticipated support from neighbors and functional status were collected from respondents at the same point in time. Because of this, it is impossible to determine whether the reported cross-sectional association between these constructs exists because one's level of anticipated support from neighbors affects one's functioning, as the theoretical foundation for this study proposes, or alternatively, whether this association exists because one's functional status affects one's level of anticipated support from neighbors. Although a good deal of longitudinal data support the idea that social relationship variables are antecedent to physical functioning (Avlund et al. 2004; Giles et al. 2004; Stuck et al. 1999), it is also plausible that functional disability results in less social engagement and ultimately leads to reductions in anticipated support from certain social ties (Carstensen 1991).

And finally, although the results of the current study are intriguing in that they document a positive association between anticipated support from neighbors and physical functioning in older age, the theoretical pathways by which this process occurs have not yet been fully tested. The current study provides some evidence that personal control beliefs partly mediate the association between anticipated support from neighbors and physical functioning. A logical next step would be to test a more specific theoretical pathway. For example, with proper measures, one could adequately test the notion that anticipated support from neighbors enhances one's efficacy to perform specific daily activities, which in turn increases levels of physical activity and social participation that can ultimately improve functioning. 
Notwithstanding these limitations, the current study should help to lay the groundwork for future research on the health benefits of support from neighbors. Replication of the current findings with more sophisticated measures and longitudinal data would provide strong confirmation that older adults need and benefit from not just intimate social ties but also social ties that offer the advantages of geographic proximity, such as is the case with neighbors. Eventually, this line of research may stimulate the development of new strategies for promoting successful aging involving the organization of social ties and support networks within neighborhoods, as well as education to increase awareness of available sources of support within one's neighborhood.

\section{NOTE}

1. Because of uncertainty about the reliability of the single-item measure of anticipated support from neighbors, supplementary analyses were conducted using perceived neighborhood quality as the major independent variable. This four-item scale uses the concept of anticipated support from neighbors as one indicator of a closely related but slightly broader concept pertaining to neighborhood cohesiveness. The other items of this scale ask respondents about perceived neighborhood safety and trust among neighbors. This broader scale has the advantage of proven reliability ( $\alpha=.68$; see Keyes 1998); however, because the current study focuses on the effects of the narrower concept of perceived availability of assistance from neighbors, the study's main analyses make use of the precision of just the single item rather than the complete scale. Despite the concerns that a single-item measure raises, it should be noted that the findings from the supplementary analyses largely support the findings from the study's main analyses. In particular, associations between perceived neighborhood quality and functional limitations follow the same pattern as the associations between anticipated support from neighbors and functional limitations, yet the effect sizes tend to be slightly larger in the supplementary analyses (a table presenting these associations is available upon request from the author). These findings not only provide evidence for an association between one's perceptions of general neighborhood quality and functional status but should also help to substantiate the main study findings by allaying concerns stemming from the use of a single-item independent variable.

\section{REFERENCES}

Aiken, Leona S. and Stephen G. West. 1991. Multiple Regression: Testing and Interpreting Interactions. Newbury Park, CA: Sage.

Avlund, Kristen, Rikke Lund, Bjorn E. Holstein, and Pernille Due. 2004. "Social Relations as Determinant of Onset of Disability in Aging." Archives of Gerontology and Geriatrics 38:8599. 
Balfour, Jennifer L. and George A. Kaplan. 2002. "Neighborhood Environment and Loss of Physical Function in Older Adults: Evidence From the Alameda County Study." American Journal of Epidemiology 155:507-15.

Baltes, Margret M. 1996. The Many Faces of Dependency in Old Age. New York: Cambridge University Press.

Baltes, Margret M. and Laura L. Carstensen. 1999. "Social-Psychological Theories and Their Applications to Aging: From Individual to Collective." Pp. 209-26 in Handbook of Theories of Aging, edited by L. Vern Bengtson and K. Warner Schaie. New York: Springer.

Brim, Orville G., Paul B. Baltes, Larry L. Bumpass, Paul D. Cleary, David L. Featherman, William R. Hazzard, et al. 1996. National Survey of Midlife Development in the United States (MIDUS), 1995-1996. ICPSR version. Ann Arbor, MI: Datastat, Inc./Boston: Harvard Medical School, Department of Health Care Policy [producers]. Ann Arbor, MI: Inter-University Consortium for Political and Social Research [distributor].

Burkhauser, Richard V., Barbara B. Butrica, and Michael J. Wasylenko. 1995. "Mobility Patterns of Older Homeowners: Are Older Homeowners Trapped in Distressed Neighborhoods?" Research on Aging 17:363-84.

Cannuscio, Carolyn, Jason Block, and Ichiro Kawachi. 2003. "Social Capital and Successful Aging: The Role of Senior Housing." Annals of Internal Medicine 139:395-99.

Cantor, Marjorie H. 1979. "Neighbors and Friends: An Overlooked Resource in the Informal Support System.” Research on Aging 1:434-63.

Carpenter, Bruce N. 1993. "Relational Competence.” Pp. 1-28 in Advances in Personal Relationships, edited by Daniel Perlman and Warren H. Jones. London: Jessica Kingsley.

Carstensen, Laura L. 1991. "Selectivity Theory: Social Activity in Life Span Context." Review of Gerontology and Geriatrics 11:195-217.

Eckenrode, John and Elaine Wethington. 1990. "The Process and Outcome of Mobilizing Social Support.” Pp. 83-103 in Personal Relationships and Social Support, edited by Steve Duck. Newbury Park, CA: Sage.

Eng, Patricia M., Eric B. Rimm, Garrett Fitzmaurice, and Ichiro Kawachi. 2002. "Social Ties and Change in Social Ties in Relation to Subsequent Total and Cause-Specific Mortality and Coronary Heart Disease Incidence in Men.” American Journal of Epidemiology 155:700-709.

Everard, Kelly M., Helen W. Lach, Edwin B. Fisher, and M. Carolyn Baum. 2000. "Relationship of Activity and Social Support to the Functional Health of Older Adults." Journal of Gerontology: Social Sciences 55B:S208-12.

Fischer, Claude S. 1982. To Dwell Among Friends. Chicago: University of Chicago Press.

Gauthier, Anne H. and Timothy M. Smeeding. 2003. "Time Use at Older Ages: Cross-National Differences." Research on Aging 25:247-74.

Giles, Lynne C., Patricia A. Metcalf, Gary F.V. Glonek, Mary A. Luszcz, and Gary R. Andrews. 2004. "The Effects of Social Networks on Disability in Older Australians." Journal of Health and Aging 16:517-38.

Gill, Diane L., Betty C. Kelley, Kathleen Williams, and Jeffrey J. Martin. 1994. "The Relationship of Self-Efficacy and Perceived Well-Being to Physical Activity and Stair Climbing in Older Adults." Research Quarterly for Exercise and Sport 65:367-71.

Katz, Sidney, Amasa B. Ford, Roland W. Moskowitz, Beverly A. Jackson, and Marjorie W. Jaffe. 1963. "Studies of Illness in the Aged. The Index of ADL: A Standardized Measure of Biological and Psychosocial Function." JAMA 21:914-19.

Kawachi, Ichiro and Lisa F. Berkman. 2003. Neighborhoods and Health. New York: Oxford University Press.

Keyes, Corey Lee M. 1998. “Social Well-Being.” Social Psychology Quarterly 1:121-37.

Krause, Neal. 1997. "Anticipated Support, Received Support, and Economic Stress Among Older Adults." Journal of Gerontology: Pscyhological Sciences 52:P284-93. 
2001. "Social Support." Pp. 272-94 in Handbook of Aging and the Social Sciences, edited by Robert H. Binstock and Linda K. George. San Diego, CA: Academic Press.

Krout, John A. 1988. "Rural Versus Urban Differences in Elderly Parents' Contact With their Children." The Gerontologist 28:198-203.

Litwak, Eugene. 1985. Helping older people. London: Guilford.

Mendes de Leon, Carlos F., Teresa E. Seeman, Dorothy I. Baker, Emily D. Richardson, and Mary E. Tinetti. 1996. "Self-Efficacy, Physical Decline, and Change in Functioning in CommunityLiving Elders: A Prospective Study." Journal of Gerontology: Social Sciences 51:S183-90.

Nagi, Saad Z. 1976. "An Epidemiology of Disability Among Adults in the United States." Milbank Memorial Quarterly Fund: Health and Society 54:439-67.

Pearlin, Leonard and Carmi Schooler. 1978. "The Structure of Coping." Journal of Health and Social Behavior 19:2-21.

Pillemer, Karl and Nina Glasgow. 2000. "Social Integration and Aging: Background and Trends." Pp. 19-47 in Social Integration in the Second Half of Life, edited by Karl Pillemer, Phyllis Moen, Elaine Wethington, and Nina Glasgow. Baltimore, MD: Johns Hopkins University Press.

Rodin, Judith. 1990. "Control by Any Other Name: Definitions, Concepts, and Processes." Pp. 117 in Self-Directedness: Cause and Effects Throughout the Life Course, edited by Judith Rodin and Carmi Schooler. Hillsdale, NJ: Lawrence Erlbaum.

Rook, Karen and Tonya L. Schuster. 1996. "Compensatory Processes in the Social Networks of Older Adults." Pp. 219-48 in Handbook of Social Support and the Family, edited by Gregory R. Pierce, Barbara R. Sarason, and Irwin G. Sarason. New York: Plenum.

Rosow, Irving and Neil Breslau. 1966. "A Guttman Health Scale for the Aged." Journal of Gerontology 21:556-59.

Ross, Catherine E. and John Mirowsky. 2002. "Family Relationships, Social Support and Subjective Life Expectancy." Journal of Health and Social Behavior 43:469-89.

Ross, Catherine E., John Mirowsky, and Karen Goldsteen. 1990. "The Impact of the Family on Health: The Decade in Review." Journal of Marriage and the Family 52:1059-78.

Sampson, Robert J. 2003. "Neighborhood-Level Context and Health: Lessons From Sociology." Pp. 132-46 in Neighborhoods and Health, edited by Ichiro Kawachi and Lisa F. Berkman. New York: Oxford University Press.

Seeman, Teresa E., Tina M. Lusignolo, and Marilyn Albert. 2001. "Social Relationships, Social Support, and Patterns of Cognitive Aging in Healthy, High Functioning Older Adults: MacArthur Studies of Successful Aging." Health Psychology 20:243-55.

Shaw, Benjamin A. and Mary Janevic. 2004. "Associations Between Anticipated Support, Physical Functioning, and Education Level Among a Nationally Representative Sample of Older Adults." Journal of Aging and Health 16:539-61.

Silverstein, Merril, Xuan Chen, and Kenneth Heller. 1996. "Too Much of a Good Thing? Intergenerational Social Support and the Psychological Well-Being of Older Parents." Journal of Marriage and the Family 58:970-82.

Simonsick, Eleanor M., Mary E. Lafferty, Caroline L. Phillips, Carlos F. Mendes de Leon, Stanislav V. Kasl, Teresa E. Seeman, Gerda Fillenbaum, Patricia Hebert, and Jon H. Lemke. 1993. "Risk Due to Inactivity in Physically Capable Older Adults." American Journal of Epidemiology 83:1443-50.

Stuck, Andreas E., Jutta M. Walthert, Thorsten Nikolaus, Christophe J. Bula, Christoph Hohmann, and John C. Beck. 1999. "Risk Factors for Functional Status Decline in CommunityLiving Elderly People: A Systematic Literature Review." Social Science and Medicine 48:445-69.

Wethington, Elaine and Allison Kavey. 2000. "Neighboring as a Form of Social Integration and Support." Pp. 190-210 in Social Integration in the Second Half of Life, edited by Karl 
Pillemer, Phyllis Moen, Elaine Wethington, and Nina Glasgow. Baltimore: Johns Hopkins University Press.

Wethington, Elaine and Ronald C. Kessler. 1986. "Perceived Support, Received Support, and Adjustment to Stressful Life Events." Journal of Health and Social Behavior 27:78-89.

Benjamin A. Shaw, Ph.D., M.P.H., is an assistant professor in the Department of Health Policy, Management and Behavior at the State University of New York at Albany School of Public Health. His research focuses on social and psychosocial determinants of health among the elderly. 